\title{
No benefit of chemotherapy in Osimertinib-treated postoperative non-small cell lung cancer patients
}

\author{
Feng $\mathrm{Li}^{1}$, Yi Zhao ${ }^{1}$, Ran Zhong ${ }^{1}$, Xiuyu Cai ${ }^{2}$, Jun Liu ${ }^{1}$, Jianxing $\mathrm{He}^{1}$, Wenhua Liang ${ }^{1}$ \\ ${ }^{1}$ China State Key Laboratory of Respiratory Disease \& National Clinical Research Center for Respiratory Disease, the First Affiliated Hospital of \\ Guangzhou Medical University, Guangzhou, China; ${ }^{2}$ Sun Yat-sen University Cancer Centre, State Key Laboratory of Oncology in South China, \\ Collaborative Innovation Centre for Cancer Medicine, Guangzhou, China \\ Correspondence to: Wenhua Liang, MD; Jianxing He, MD. Department of Thoracic Surgery and Oncology, the First Affiliated Hospital of Guangzhou \\ Medical University; Guangzhou Institute of Respiratory Disease \& China State Key Laboratory of Respiratory Disease, No. 151 Yanjiang Road, \\ Guangzhou 510120, China. Email: liangwh1987@163.com; drjianxing.he@gmail.com.
}

Submitted Aug 1, 2021. Accepted for publication Aug 22, 2021.

doi: $10.21037 /$ tlcr-21-640

View this article at: https://dx.doi.org/10.21037/tlcr-21-640

In ADAURA, Osimertinib showed a significant disease-free survival benefit in resected epidermal growth factor receptor (EGFR)-mutated non-small cell lung cancer (NSCLC) patients with or without adjuvant chemotherapy (1). However, the necessity of chemotherapy in the Osimertinibbased adjuvant therapy is still in hot debate, and no related randomized controlled trials exist or being planned. Therefore, by re-analyzing the data of ADAURA (2), we developed a cross-arm comparison of adjuvant chemotherapy versus non-adjuvant chemotherapy patients in Osimertinib and placebo group respectively, to explore the value of adjuvant chemotherapy indirectly.

We extracted Kaplan-Meier survival curves from ADAURA study (2), then separated the curves of adjuvant chemotherapy arm and non-adjuvant chemotherapy arm from Osimertinib group. Engauge Digitizer (4.1) was used to read the Kaplan-Meier curves and extract all horizontal and vertical coordinates for curve reconstruction. Specifically, the extracted curves were combined to obtain the survival curve plot of adjuvant chemotherapy versus non-adjuvant chemotherapy in Osimertinib group. Similar method was used to extract and redraw the survival curve plot of adjuvant chemotherapy versus non-adjuvant chemotherapy in placebo group. We used numbers at risk, number of events, and numbers censored during each segmented interval to generate the overall hazard ratio (HR) and associated statistics, according to the methods described by Tierney (3). Considering that some patients received adjuvant chemotherapy before randomization,
3 months (median chemotherapy time) was superimposed in the chemotherapy group in sensitivity analyses.

In the placebo group (Figure 1), patients with stage IIIIIA appeared to benefit from adjuvant chemotherapy [HR, 0.77; $95 \%$ confidence interval (CI), $0.42-1.40$ in patients with stage II; HR, 0.61; 95\% CI, 0.35-1.07 in patients with stage IIIA], while patients with stage IB did not (HR, 1.44; 95\% CI, 0.63-3.28). An upward trend in benefit was observed with the stage increased, which resembled to LACE (4) study. In the Osimertinib group (Figure 2), no benefit of adjuvant chemotherapy was presented in all disease stage (HR, 1.99; 95\% CI, 0.48-8.26 in patients with stage IB; HR, 0.48 ; $95 \% \mathrm{CI}, 0.13-1.80$ in patients with stage II; HR, 1.06 ; $95 \%$ CI, $0.27-4.12$ in patients with stage IIIA). In sensitivity analysis, 3 months was superimposed in the chemotherapy group to adjust the chemotherapy time. The results were consistent with the primary analyses.

We further tried to explore why adding adjuvant chemotherapy was not beneficial among Osimertinib-based patients. The prevalence of co-occurring genetic alterations contributed to the early resistance in patients treated with EGFR-tyrosine kinase inhibitor (TKI) alone, and TKI combined chemotherapy might delay the resistance (5). Previous study showed that among all co-occurring mutated genes, only cell cycle gene alteration may drive primary resistance to Osimertinib (6). Therefore, we explored the cell cycle gene co-mutation frequency in stage IB to IIIA EGFR-mutated NSCLC patients and found that only 
Stage IB to IIIA
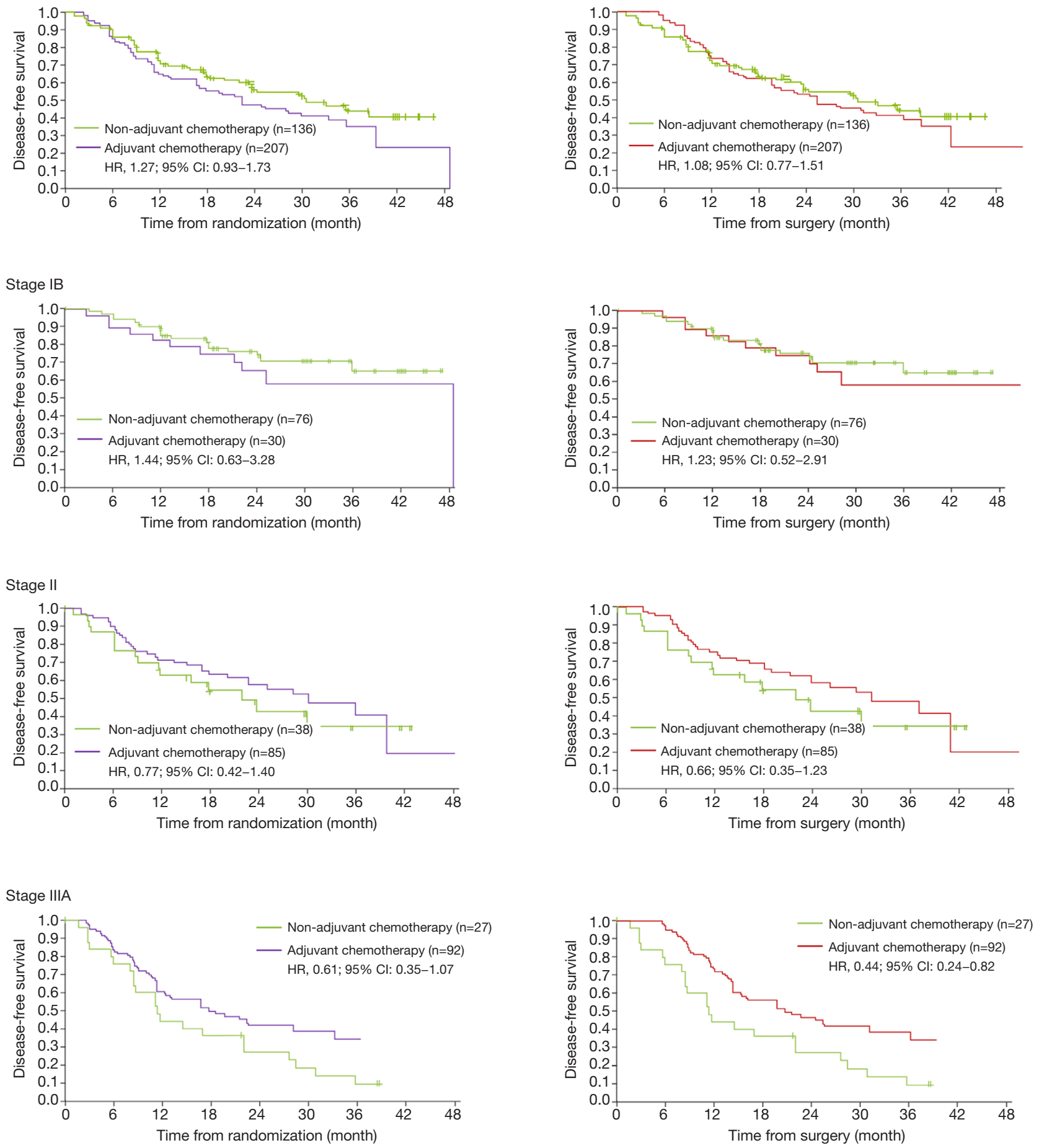

Figure 1 Kaplan-Meier curves of adjuvant chemotherapy versus non-adjuvant chemotherapy in placebo group. Patients with stage II-IIIA benefited from adjuvant chemotherapy, while stage IB patients did not. The Kaplan-Meier curves plots in the left represent the primary analysis of adjuvant chemotherapy versus non-adjuvant chemotherapy group. The Kaplan-Meier curves plots in the right represent the sensitivity analysis of adjuvant chemotherapy versus non-adjuvant chemotherapy group. HR, hazard ratio; CI, confidence interval. 
Stage IB to IIIA
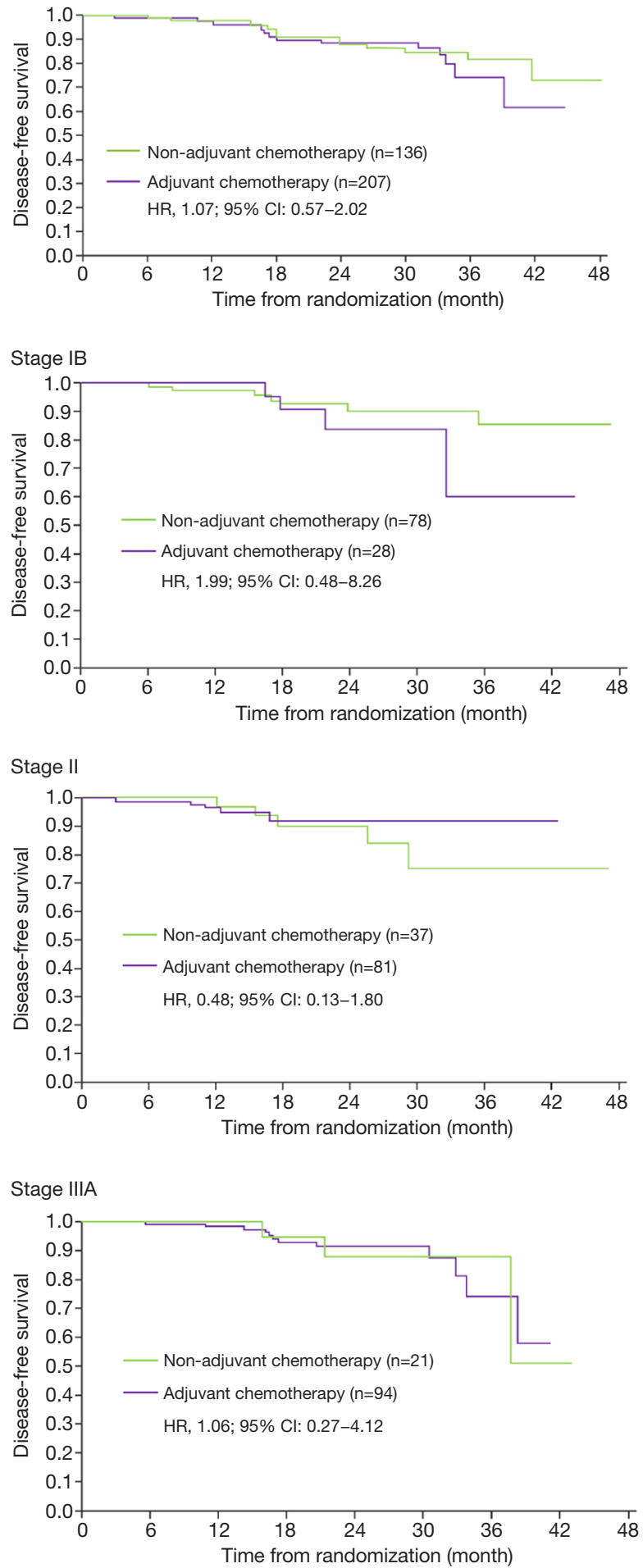
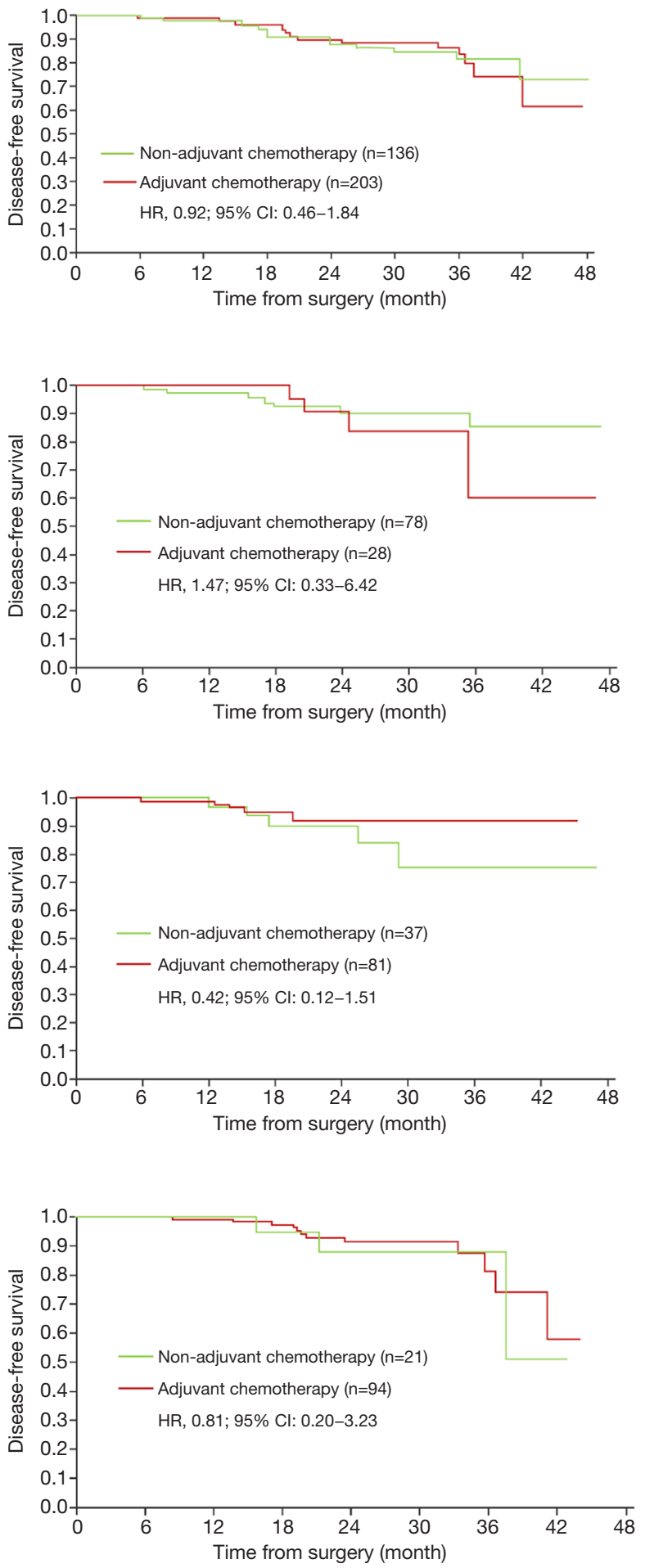

Figure 2 Kaplan-Meier curves of adjuvant chemotherapy versus non-adjuvant chemotherapy in Osimertinib group. No benefit is noted in patients received adjuvant chemotherapy, stratified according to disease stage. The Kaplan-Meier curves plots in the left represent the primary analysis of adjuvant chemotherapy versus non-adjuvant chemotherapy group. The Kaplan-Meier curves plots in the right represent the sensitivity analysis of adjuvant chemotherapy versus non-adjuvant chemotherapy group. HR, hazard ratio; CI, confidence interval. 

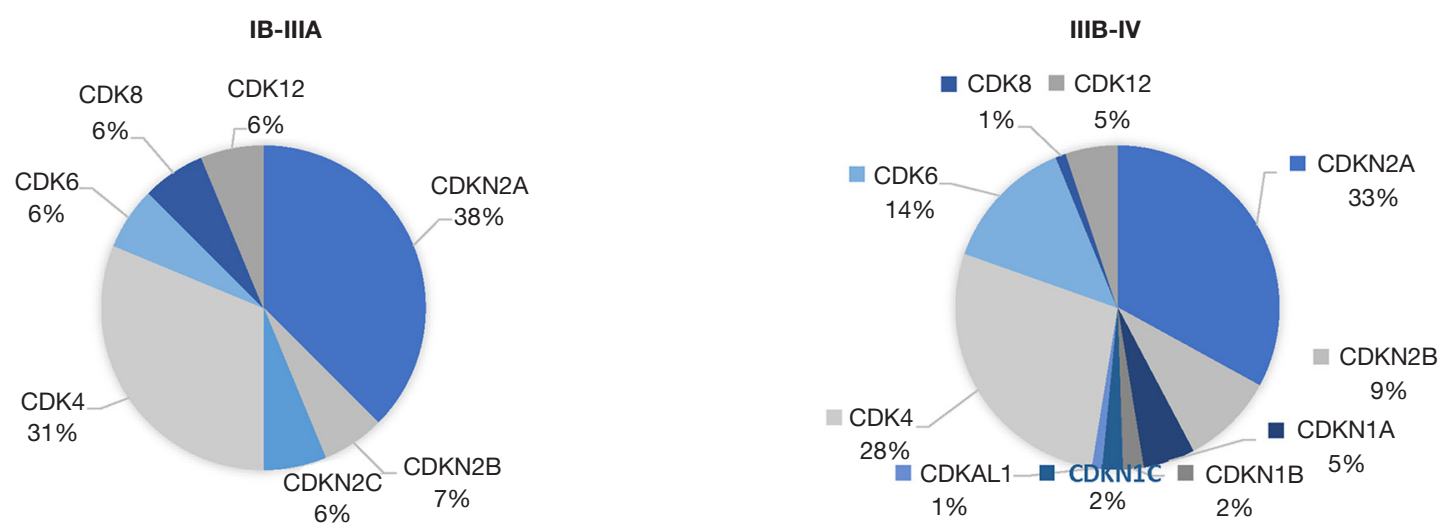

Figure 3 The frequency of co-mutated cell cycle gene subtype in postoperative EGFR-mutated NSCLC patients. EGFR, epidermal growth factor receptor; NSCLC, non-small cell lung cancer; CDK, cyclin-dependent kinase.

$13.3 \%(16 / 120)$ cases were positive, which was lower than that in stage IIIB-IV patients $(25.5 \%, 97 / 381)$, based on multigene-profiling data of the first affiliated hospital of Guangzhou Medical University from January 2018 to January 2021 (Figure 3).

We observed no benefit of chemotherapy in postoperative NSCLC patients received Osimertinib according to crossarm comparison of ADAURA study. The co-mutated cell cycle gene which limits Osimertinib response was relatively rare in resected NSCLC, thus the efficacy of Osimertinib monotherapy may be significant enough for most patients. A Osimertinib-based, chemotherapy-free adjuvant regimen may be available and preferable for postoperative NSCLC patients with EGFR mutation. Although this comparison was limited by its post-hoc manner and the potential biases from non-randomization setting, it encourages prospective studies to clarify the value of chemotherapy in patients receiving Osimertinib-based adjuvant therapy.

\section{Acknowledgments}

Funding: None.

\section{Footnote}

Provenance and Peer Review: This article was a standard submission to the journal. The article did not undergo external peer review.

Conflicts of Interest: All authors have completed the ICMJE uniform disclosure form (available at https://dx.doi. org/10.21037/tlcr-21-640). WL serves as an unpaid
Associate Editor-in-Chief of Translational Lung Cancer Research. The other authors have no conflicts of interest to declare.

Ethical Statement: The authors are accountable for all aspects of the work in ensuring that questions related to the accuracy or integrity of any part of the work are appropriately investigated and resolved.

Open Access Statement: This is an Open Access article distributed in accordance with the Creative Commons Attribution-NonCommercial-NoDerivs 4.0 International License (CC BY-NC-ND 4.0), which permits the noncommercial replication and distribution of the article with the strict proviso that no changes or edits are made and the original work is properly cited (including links to both the formal publication through the relevant DOI and the license). See: https://creativecommons.org/ licenses/by-nc-nd/4.0/.

\section{References}

1. Wu YL, Tsuboi M, He J, et al. Osimertinib in Resected EGFR-Mutated Non-Small-Cell Lung Cancer. N Engl J Med 2020;383:1711-23.

2. Wu Y, John T, Grohe C, et al. OA06.04 Postoperative Chemotherapy Use and Outcomes from ADAURA: Osimertinib as Adjuvant Therapy for Resected EGFR Mutated NSCLC. J Thorac Oncol 2021;16:S113-4.

3. Tierney JF, Stewart LA, Ghersi D, et al. Practical methods for incorporating summary time-to-event data into metaanalysis. Trials 2007;8:16. 
4. Pignon JP, Tribodet H, Scagliotti GV, et al. Lung adjuvant cisplatin evaluation: a pooled analysis by the LACE Collaborative Group. J Clin Oncol 2008;26:3552-9.

5. Hosomi Y, Morita S, Sugawara S, et al. Gefitinib Alone Versus Gefitinib Plus Chemotherapy for NonSmall-Cell Lung Cancer With Mutated Epidermal

Cite this article as: Li F, Zhao Y, Zhong R, Cai X, Liu J, He J, Liang W. No benefit of chemotherapy in Osimertinib-treated postoperative non-small cell lung cancer patients. Transl Lung Cancer Res 2021;10(8):3689-3693. doi: 10.21037/tlcr-21-640
Growth Factor Receptor: NEJ009 Study. J Clin Oncol 2020;38:115-23.

6. Blakely CM, Watkins TBK, $\mathrm{Wu} \mathrm{W}$, et al. Evolution and clinical impact of co-occurring genetic alterations in advanced-stage EGFR-mutant lung cancers. Nat Genet 2017;49:1693-704. 